\title{
Financial Comparisons Before and After Joining a Strategic Alliance in the Airline Industry
}

\author{
Dwi Kartikasari \\ Dept. of Business and Management \\ Politeknik Negeri Batam \\ Batam, Indonesia \\ https://orcid.org/0000-0002-3222-4426
}

\begin{abstract}
In addition to expanding the capacity of flight services, alliances or strategic cooperation between airlines throughout the world are also used as a mean to increase the revenue of their alliance members. However, it is possible that expectations for increasing revenue and financial performance cannot be realized. Therefore, this study seeks to compare financial performance of $\mathbf{1 0}$ members of the SkyTeam alliance before and after joining the alliance. Data were collected from the Osiris database for the duration of 4 years before and 4 years after the inclusion to the SkyTeam. This study indicates that airlines in the alliance on average record a low profitability and liquidity ratios as well as a high gearing ratio which makes the company be at bigger financial risk of default. More often than not, sample data are drawn from a normally distributed population. Once data are confirmed normally distributed, paired sample t-test are used, otherwise Wilcoxon test are utilized. This study shows the significance level of each test and suggests that in most of financial ratios out of 20 ratios across airlines, financial difference before and after joining the strategic alliance is not significant. This finding which shows that the alliance has not met expectations is not surprising across other economic sectors. Future studies are needed in search of factors affecting alliance performance.
\end{abstract}

Keywords-financial comparisons, strategic alliance, airline industry, SkyTeam

\section{INTRODUCTION}

In the era of globalization where competition between companies is getting tougher, all companies are trying to improve their competitiveness, including airlines. The airline business sector in Indonesia is a business sector that is less discussed from a national financial perspective because there is only one airline company whose financial statements are available to the public in Indonesia, so it is not possible for the comparative process. This not only happens in Indonesia, but also occurs in many other countries, where airlines listed on the stock exchange are very limited.

To be able to provide flight services for passengers or cargo, airlines can do either 1) rent, 2) own, or 3) form cooperation or alliances with other airlines. To operate as a commercial airline, either cargo, chartered or scheduled one, an airline must have a permit issued by the civil aviation authority in Indonesia under the Ministry of Transportation as 121 or 135 Air Operator Certificate (AOC). As of 2019, more than 50 airlines already have received this permit because they are considered to comply with Law No. 1 of 2009 concerning Aviation along with its derivative rules. The Ministry of Transportation of the Republic of Indonesia continues to improve the safety level of the aviation environment so that in 2018, all airlines in Indonesia are allowed to fly in the European sky. The Indonesian government takes part in regulating the world of aviation because of the skyrocketing growth of flights in Indonesian archipelagic countries which are predicted to reach 20 percent per year [1] and the strong economic and tourism needs supported by the aviation world.

In addition to expanding the capacity of aviation services, strategic alliances or cooperation between airlines around the world are also used as a means to be able to be exposed to the latest technology, enter new markets abroad by cutting down the bureaucracy and protection of destination countries, and learning areas from more advanced companies [2]. An airline joins the alliance as one of its growth strategies [3].

The strategic alliances of the largest airlines in the world include SkyTeam, Star Alliance, and Oneworld. All three are called mega alliances because they consist of various companies from diverse parts of the world [4]. This alliance does varying publicity to attract customers so as to increase the income of its alliance members. However, due to the quite obvious differences between alliance members, it is possible that expectations for increasing income cannot be realized or that the increase in income from alliances is not worth the cost of alliance membership. Therefore, this study seeks to see whether there are significant differences of the financial performance of airlines in years before and after joining the strategic alliance.

The author hopes that if the series of studies on the impact of the alliance on the aviation industry is proven to be able to increase load factors, increase aircraft utilities, and improve the company's financial performance, the strategic alliance can be utilized by the majority of airlines that are not currently incorporated in the alliance. Aviation stock investors who currently suffer from small yields and high fluctuations [5] can be better compensated when airline management improves.

\section{LITERATURE REVIEW}

There is no relationship between financial performance of airlines with different business models, regardless low cost 
carriers or full service carriers [6]. In the case of airline strategic alliances, alliance membership in the short term does not significantly influence the quality of airline services [7]. Consumers still choose airline brands individually without regard to their affiliates with strategic alliances [8]. Thus, if consumers still focus on the airline brand individually and not their alliance brand, then strategic alliance management needs to carry out various strategies to maintain airline membership in its alliance by offering various other benefits [9].

When comparing data before and after the joining of Garuda Indonesia into an alliance (Skyteam), it is argued that the number of passengers rose 60 percent, revenue rose 50 percent, where the contribution of income through alliances was 0.3 percent and the cost ratio was still around 3 percent so it could be concluded that the strategic alliances carried out by Garuda Indonesia had provided positive benefits for the company, although not too big, but the potential is supposed to be even greater in the future [10]. Nevertheless, there are no studies that specifically prove changes in the overall financial performance of all members of the strategic alliance.

The financial ratios used as proxy for financial performance followed by their definitions are [11]:

TABLE I. INDUSTRIAL FINANCIAL RATIOS [11]

\begin{tabular}{|c|c|c|c|}
\hline$\#$ & $\begin{array}{l}\text { Account } \\
\text { Number }\end{array}$ & Ratios & Definitions \\
\hline \multicolumn{4}{|c|}{ Profitability Ratios } \\
\hline 1 & 31005 & Profit Margin (\%) & $\begin{array}{l}\text { P/L Before tax / Operating Revenue } \\
\text { (Turnover) * } 100 \\
(30260 / 30205) * 100\end{array}$ \\
\hline 2 & 31010 & \begin{tabular}{|l|} 
Return on \\
Shareholders \\
Funds $(\%)$
\end{tabular} & $\begin{array}{l}\text { (P/L Before tax / Shareholders Funds) } \\
* 100 \\
(30260 / 30090) * 100\end{array}$ \\
\hline 3 & 31015 & $\begin{array}{l}\text { Return on Total } \\
\text { Assets }(\%)\end{array}$ & $\begin{array}{l}\text { (P/L Before tax / Total Assets }) * 100= \\
(30260 / 30050) * 100\end{array}$ \\
\hline 4 & 31020 & $\begin{array}{l}\text { Return on Capital } \\
\text { Employed }(\%)\end{array}$ & $\begin{array}{l}\text { (P/L Before tax Interest Expense) / } \\
\text { (Shareholders Funds }+ \text { Non-Current } \\
\text { Liabilities }) * 100=(30260-30245) / \\
(30090+30075) * 100\end{array}$ \\
\hline 5 & 31045 & $\begin{array}{l}\text { Cash Flow/ } \\
\text { Operating } \\
\text { Revenue (\%) }\end{array}$ & $\begin{array}{l}\text { (Cash Flow / Operating Revenue) } * \\
100 \\
(30295 / 30205) * 100\end{array}$ \\
\hline 6 & 31050 & Gross Margin (\%) & $\begin{array}{l}\text { (Gross Profit } / \text { Operating Revenue }) * \\
100=(30220 / 30205) * 100\end{array}$ \\
\hline 7 & 31055 & EBIT Margin (\%) & $\begin{array}{l}(\text { EBIT / Operating Revenue }) * 100= \\
(30310 / 30205) * 100\end{array}$ \\
\hline 8 & 31060 & $\begin{array}{l}\text { EBITDA Margin } \\
(\%)\end{array}$ & $\begin{array}{l}\text { (EBITDA / Operating Revenue }) * 100 \\
=(30320 / 30205) * 100\end{array}$ \\
\hline 9 & 31065 & $\operatorname{ROE}(\%)$ & $\begin{array}{l}\text { (P/L for period / Shareholders Funds) } \\
* 100 \\
(30280 / 30090) * 100\end{array}$ \\
\hline 10 & 31070 & ROA (\%) & $\begin{array}{l}(\mathrm{P} / \mathrm{L} \text { for period } / \text { Total Assets }) * 100= \\
(30280 / 30050) * 100\end{array}$ \\
\hline 11 & 31075 & ROCE (\%) & $\begin{array}{l}\text { [(P/L for period - Interest Expense) } / \\
\text { (Shareholders Funds + Non-Current } \\
\text { Liabilities })] * 100=[(3028030245) / \\
(30090+30075)] * 100\end{array}$ \\
\hline \multicolumn{4}{|c|}{ Liquidity Ratios } \\
\hline 12 & 31105 & Current ratio & $\begin{array}{l}\text { Current Assets / Current Liabilities = } \\
30005 / 30055\end{array}$ \\
\hline 13 & 31110 & Liquidity ratio & \begin{tabular}{|l} 
(Current Assets Stocks) $/$ Current \\
Liabilities $=(30005-30010) / 30055$
\end{tabular} \\
\hline 14 & 31115 & Interest Cover & $\begin{array}{l}\text { (Operating P/L / Interest Expense) } *-1 \\
=(30235 / 30245) *-1\end{array}$ \\
\hline 15 & 31120 & Collection Period & (Debtors / Operating Revenue) $* 360$ \\
\hline
\end{tabular}

\begin{tabular}{|c|c|l|l|}
\hline$\#$ & $\begin{array}{c}\text { Account } \\
\text { Number }\end{array}$ & \multicolumn{1}{|c|}{ Ratios } & \multicolumn{1}{c|}{ Definitions } \\
\hline & & (days) & $=(30015 / 30205) * 360$ \\
\hline 16 & 31125 & $\begin{array}{l}\text { Credit Period } \\
\text { (days) }\end{array}$ & $\begin{array}{l}\text { (Creditors / Operating Revenue) *360 } \\
=(30065 / 30205) * 360\end{array}$ \\
\hline 17 & 31220 & Stock Turnover & $\begin{array}{l}\text { Operating Revenue / Stocks } \\
30205 / 30010\end{array}$ \\
\hline 18 & 31225 & $\begin{array}{l}\text { Net Assets } \\
\text { Turnover }\end{array}$ & $\begin{array}{l}\text { Operating Revenue / (Shareholders } \\
\text { Funds + Noncurrent } \\
\text { Liabilities) = 30205 / (30090 + 30075) }\end{array}$ \\
\hline \multicolumn{5}{|c|}{ Structure ratios } \\
\hline 19 & 31310 & Solvency ratio (\%) & $\begin{array}{l}\text { (Shareholders Funds / Total Assets) * } \\
100=(30090 / 30050) * 100\end{array}$ \\
\hline 20 & 31315 & Gearing (\%) & $\begin{array}{l}\text { (Non-current Liabilities + Loans) / } \\
\text { Shareholders Funds } \\
\text { (30075 + 30060) / 30090 }\end{array}$ \\
\hline
\end{tabular}

All ratios are selected based on availability criteria in the period of the research. In this study, the data is provided by the Osiris system.

\section{METHODOLOGY}

In this study, the SkyTeam alliance was chosen because this alliance was the world's largest alliance in terms of passenger numbers in 2016 (Skyteam.com). Although it is the youngest alliance, this alliance is the alliance with the fastest growth compared to other alliances, so several times it was chosen as the Air Transport Awards' airline alliance of the year. In addition, this alliance is the only alliance that entered Indonesia through Garuda Indonesia, the youngest member to join in 2014 , so the authors hope that research on this alliance will benefit the national aviation industry.

As of 2019, SkyTeam has 19 members, but 9 of its members are not public companies or their data are not available online or their data are not completed yet. Therefore, in this study, the author only used 10 airlines, including Air France and KLM separately. The research period is 4 years before and 4 years after the inclusion to the SkyTeam as shown in table below.

TABLE II. SAMPLE AIRLINES

\begin{tabular}{|r|l|l|l|c|l|}
\hline$\#$ & $\begin{array}{l}\text { Member } \\
\text { airline }\end{array}$ & Country & $\begin{array}{l}\text { Join (DD- } \\
\text { MM-YY) }\end{array}$ & $\begin{array}{c}\text { Research } \\
\text { Years }\end{array}$ & Remarks \\
\hline 1 & Aeroflot & Russia & $14-04-06$ & $2002-2009$ & Included as sample \\
\hline 2 & $\begin{array}{l}\text { Aerolíneas } \\
\text { Argentinas }\end{array}$ & Argentina & $29-08-12$ & $2008-2015$ & Private \\
\hline 3 & Aeroméxico & Mexico & $22-06-00$ & $1996-2003$ & $\begin{array}{l}\text { No data available until } \\
2002\end{array}$ \\
\hline 4 & Air Europa & Spain & $04-09-07$ & $2003-2010$ & Private \\
\hline 5 & Air France & France & $22-06-00$ & $1996-2003$ & Included as sample \\
\hline 6 & Alitalia & Italy & $13-01-09$ & $2005-2012$ & Privatization in 2008 \\
\hline 7 & China Airlines & Taiwan & $28-09-11$ & $2007-2014$ & Included as sample \\
\hline 8 & $\begin{array}{l}\text { China Eastern } \\
\text { Airlines }\end{array}$ & China & $21-06-11$ & $2007-2014$ & Included as sample \\
\hline 9 & $\begin{array}{l}\text { Czech } \\
\text { Airlines }\end{array}$ & $\begin{array}{l}\text { Czech } \\
\text { Republic }\end{array}$ & $25-03-01$ & $1997-2004$ & Private \\
\hline 10 & $\begin{array}{l}\text { Delta Air } \\
\text { Lines }\end{array}$ & United States & $22-06-00$ & $1996-2003$ & Included as sample \\
\hline 11 & $\begin{array}{l}\text { Garuda } \\
\text { Indonesia }\end{array}$ & Indonesia & $05-03-14$ & $2010-2017$ & Included as sample \\
\hline 12 & $\begin{array}{l}\text { Kenya } \\
\text { Airways }\end{array}$ & Kenya & $04-09-07$ & $2003-2010$ & Included as sample \\
\hline 13 & KLM & Netherlands & $13-09-04$ & $2000-2007$ & Included as sample \\
\hline 14 & Korean Air & South Korea & $22-06-00$ & $1996-2003$ & Included as sample \\
\hline 15 & $\begin{array}{l}\text { Middle East } \\
\text { Airlines }\end{array}$ & Lebanon & $28-06-12$ & $2008-2015$ & Private \\
\hline
\end{tabular}




\begin{tabular}{|r|l|l|l|l|l|}
\hline 16 & Saudia & Saudi Arabia & $29-05-12$ & $2008-2015$ & Private \\
\hline 17 & TAROM & Romania & $25-06-10$ & $2006-2013$ & Private \\
\hline 18 & $\begin{array}{l}\text { Vietnam } \\
\text { Airlines }\end{array}$ & Vietnam & $10-06-10$ & $2006-2013$ & $\begin{array}{l}\text { Private but go public } \\
\text { afterward }\end{array}$ \\
\hline 19 & XiamenAir & China & $21-11-12$ & $2008-2015$ & Included as sample \\
\hline
\end{tabular}

The last member is Garuda Indonesia that joined SkyTeam in 14 so its research period ends in 17 because the international financial database has not provided data in 18 in a complete form. The companies that consistently join as long as Garuda Indonesia joins the alliance are Aeroflot, AirFrance, KLM, China Airlines, China Eastern, Delta Airlines, Garuda Indonesia, Kenya Airways, Korean Air, and Xiamen Air. All financial statements expire in December, except Kenya which ends in March. Thus there are 80 data observed per financial indicator with indicators used so that there are a total of 1600 data.

Data are collected from the international financial database Osiris Bureau van Dijk via the link https://wrdsweb.wharton.upenn.edu/wrds/index.cfm provided by Wharton Reserach Data Services with paid membership. Data is processed using Microsoft Excel for descriptive statistics and SPSS for normality test dan paired difference test. When data are normally distributed, paired sample t-test are implemented, otherwise Wilcoxon test are used. Then the data were analyzed descriptively qualitatively and enriched with other online news sources.

\section{RESULTS AND DISCUSSIONS}

\section{A. Descriptive Statistics}

Table below shows that airlines in the alliance on average record a low profitability ratio as can be seen from profit margin at around 5 percent and ROA at approximately 2 percent. The results of this study are supported by a claim that the air transportation industry often records the lowest ROI compared to other industrial sectors [12]. The thinness of all profitability performance is caused by the lack of income generated by the aviation industry. The main reason for the lack of profits is the difficulty of raising revenue because the ticket price war is accompanied by high costs such as fuel costs, labor costs, airport fares, and insurance [12].
TABLE III. DESCRIPTIVE STATISTICS

\begin{tabular}{|r|l|c|c|r|r|}
\hline$\#$ & \multicolumn{1}{|c|}{ Ratios } & Mean & $\begin{array}{c}\text { Std. } \\
\text { Deviation }\end{array}$ & Min & Max \\
\hline 1 & Profit Margin (\%) & 5,84 & 3,11 & 0,67 & 9,18 \\
\hline 2 & Return on Shareholders Funds & 7,23 & 10,95 & $-10,81$ & 21,86 \\
\hline 3 & Return on Total Assets (\%) & 3,86 & 2,17 & 1,47 & 6,83 \\
\hline 4 & Return on Capital Employed & 7,40 & 3,70 & 1,41 & 12,26 \\
\hline 5 & Cash Flow/ Operating Revenue & 13,92 & 1,45 & 11,59 & 16,06 \\
\hline 6 & Gross Margin (\%) & 42,81 & 2,98 & 39,57 & 48,38 \\
\hline 7 & EBIT Margin (\%) & 7,44 & 2,33 & 3,68 & 10,02 \\
\hline 8 & EBITDA Margin (\%) & 15,22 & 1,34 & 13,76 & 16,84 \\
\hline 9 & ROE (\%) & 5,23 & 9,11 & $-8,03$ & 16,94 \\
\hline 10 & ROA (\%) & 2,72 & 1,85 & 0,57 & 5,14 \\
\hline 11 & ROCE (\%) & 5,70 & 3,48 & $-0,63$ & 9,84 \\
\hline 12 & Current ratio & 1,00 & 0,21 & 0,77 & 1,25 \\
\hline 13 & Liquidity ratio & 0,92 & 0,21 & 0,7 & 1,17 \\
\hline 14 & Interest Cover & 6,85 & 10,40 & 1,6 & 32,25 \\
\hline 15 & Collection Period (days) & 32,99 & 5,95 & 27 & 44,7 \\
\hline 16 & Credit Period (days) & 27,87 & 6,71 & 20,67 & 40,33 \\
\hline 17 & Stock Turnover & 51,56 & 27,85 & 33,56 & 119,44 \\
\hline 18 & Net Assets Turnover & 1,23 & 0,16 & 1,07 & 1,5 \\
\hline 19 & Solvency ratio (\%) & 28,95 & 3,60 & 23,61 & 33,11 \\
\hline 20 & Gearing (\%) & 228,63 & 20,81 & 198,61 & 270,8 \\
\hline
\end{tabular}

Likewise, liquidity ratios are considered low at around 1 . Investors usually seek for liquidity ratio of around 2 as a safe cushion. A gearing ratio far above 50 percent at around 228 percent is typically classified as highly levered or geared. Thus, the company would be at bigger financial risk of default and bankruptcy. This results confirmed the aviation industry as a capital-intensive industry which are less popular among investors because shareholders of airlines throughout the world are not well compensated by the aviation industry [5]. Therefore, it is not surprising that stock analysts do not like airline stocks [12].

\section{B. Normality Test}

Data are tested their normality. Table below indicates that more often than not, sample data are drawn from a normally distributed population.

TABLE IV. TESTS OF NORMALITY

\begin{tabular}{|c|c|c|c|c|c|c|c|c|c|c|c|c|}
\hline$\#$ & Ratios & \begin{tabular}{|l|} 
Average \\
\end{tabular} & Aeroflot & Airfrance & China & China Eastern & Delta* & Garuda* & Kenya & KLM & Korea & Xiamen* \\
\hline 1 & Profit Margin $(\%)$ & 0,24 & 0,91 & $0,05^{\mathrm{a}}$ & $0,02^{\mathrm{a}}$ & 0,95 & 0,67 & 0,43 & 0,61 & 0,59 & 0,41 & 0,23 \\
\hline 2 & Return on Shareholders Funds & 0,85 & 0,34 & $0,00^{\mathrm{a}}$ & $0,01^{\mathrm{a}}$ & 0,44 & 0,52 & 0,35 & 0,40 & 0,37 & 0,42 & 0,88 \\
\hline 3 & Return on Total Assets (\%) & 0,17 & $0,00^{\mathrm{a}}$ & 0,13 & $0,00^{\mathrm{a}}$ & 0,52 & 0,89 & 0,48 & 0,56 & 0,58 & 0,19 & 0,53 \\
\hline 4 & Return on Capital Employed & 0,77 & 0,26 & 0,21 & $0,00^{\mathrm{a}}$ & 0,73 & 0,91 & 0,53 & 0,15 & 0,69 & 0,91 & 0,62 \\
\hline 5 & Cash Flow/ Operating Revenue & 0,99 & $0,01^{\mathrm{a}}$ & $0,02^{\mathrm{a}}$ & 0,21 & 0,63 & 0,87 & 0,93 & 0,10 & 0,11 & 0,67 & 0,89 \\
\hline 6 & Gross Margin (\%) & 0,21 & 0,51 & 0,35 & 0,69 & 0,96 & 0,52 & N/A & 0,55 & 0,40 & $0,04^{\mathrm{a}}$ & 0,53 \\
\hline 7 & EBIT Margin (\%) & 0,36 & 0,92 & 0,36 & $0,00^{\mathrm{a}}$ & 0,21 & 0,80 & 0,85 & 0,49 & 0,67 & 0,65 & 0,25 \\
\hline 8 & EBITDA Margin (\%) & 0,07 & 0,80 & 0,47 & 0,58 & 0,70 & 0,44 & 0,53 & 0,40 & 0,13 & 0,78 & 0,98 \\
\hline 9 & ROE (\%) & 0,66 & 0,59 & $0,00^{\mathrm{a}}$ & $0,01^{\mathrm{a}}$ & 0,33 & 0,58 & 0,40 & 0,88 & 0,25 & 0,20 & 0,90 \\
\hline 10 & ROA (\%) & 0,24 & $0,02^{\mathrm{a}}$ & 0,08 & $0,00^{\mathrm{a}}$ & 0,37 & 0,87 & 0,54 & 0,99 & 0,47 & 0,95 & 0,69 \\
\hline 11 & ROCE $(\%)$ & 0,63 & 0,36 & 0,14 & $0,00^{\mathrm{a}}$ & 0,58 & 0,91 & 0,39 & 0,76 & 0,53 & 0,49 & 0,68 \\
\hline 12 & Current ratio & $0,05^{\mathrm{a}}$ & 0,59 & 0,69 & 0,73 & 0,42 & 0,97 & 0,58 & $0,02^{\mathrm{a}}$ & 0,24 & 0,22 & 0,55 \\
\hline 13 & Liquidity ratio & $0,05^{\mathrm{a}}$ & 0,37 & 0,61 & 0,97 & 0,43 & 0,88 & 0,83 & $0,02^{\mathrm{a}}$ & 0,43 & 0,78 & 0,55 \\
\hline 14 & Interest Cover & $0,00^{\mathrm{a}}$ & 0,41 & 0,84 & $0,00^{\mathrm{a}}$ & 0,14 & 0,96 & 0,99 & 0,21 & 0,42 & 0,60 & N/A \\
\hline 15 & Collection Period (days) & 0,29 & 0,61 & 0,58 & 0,60 & 0,75 & 0,94 & 0,93 & 0,96 & 0,05 & 0,46 & 0,58 \\
\hline 16 & Credit Period (days) & 0,25 & $0,04^{\mathrm{a}}$ & 0,13 & 0,29 & 0,65 & N/A & 0,76 & 0,38 & 0,65 & 0,46 & 0,20 \\
\hline 17 & Stock Turnover & $0,00^{\mathrm{a}}$ & 0,56 & 0,27 & 0,19 & 0,15 & 0,82 & 0,92 & 0,76 & 0,84 & 0,57 & 1,00 \\
\hline
\end{tabular}




\begin{tabular}{|l|l|c|c|c|c|c|c|c|c|c|c|c|}
\hline 18 & Net Assets Turnover & 0,08 & 0,76 & 0,46 & $0,04^{\mathrm{a}}$ & 0,09 & 0,92 & 0,96 & 0,23 & 0,08 & 0,89 & 0,41 \\
\hline 19 & Solvency ratio (\%) & 0,27 & 0,64 & $0,03^{\mathrm{a}}$ & $0,04^{\mathrm{a}}$ & 0,27 & 0,75 & 0,47 & 0,21 & 0,34 & 0,99 & 0,72 \\
\hline 20 & Gearing (\%) & 0,46 & 0,10 & $0,00^{\mathrm{a}}$ & $0,00^{\mathrm{a}}$ & 0,25 & 0,89 & 0,80 & 0,93 & 0,17 & 0,51 & 0,96 \\
\hline Normally distributed & 16 & 16 & 14 & 8 & 20 & 19 & 19 & 18 & 20 & 19 & 19 \\
\hline Not normally distributed & 4 & 4 & 6 & 12 & 0 & 0 & 0 & 2 & 0 & 1 & 0 & 0 \\
\hline Not Available & 0 & 0 & 0 & 0 & 0 & 1 & 1 & 0 & 0 & 0 & \\
\hline
\end{tabular}

* Normality test results are obtained from different procedure (K-S) of SPSS because standard procedure does not result in any output for Shapiro Wilk test

${ }^{a}$ Not normally distributed

\section{Paired Difference Analysis}

Once data are confirmed normally distributed, paired sample t-test are used, otherwise Wilcoxon test are utilized.
Table below illustrates the significance level of each test and suggests that in most of financial ratios across airlines, financial difference before and after joining the strategic alliance is not significant.

TABLE V. PAIRED DIFFERENCE TEST

\begin{tabular}{|c|c|c|c|c|c|c|c|c|c|c|c|c|}
\hline$\#$ & Ratios & Average & Aeroflot & Airfrance & China & China Eastern & Delta* & Garuda* & Kenya & KLM & Korea & Xiamen* \\
\hline 1 & Profit Margin (\%) & 0,95 & 0,88 & 0,36 & 0,50 & 0,34 & 0,13 & 0,11 & 0,73 & 0,15 & 0,06 & 0,91 \\
\hline 2 & Return on Shareholders Funds & 0,54 & $0,03^{\mathrm{a}}$ & 0,34 & 0,48 & 0,43 & 0,29 & 0,15 & 0,54 & 0,18 & 0,83 & 0,12 \\
\hline 3 & Return on Total Assets (\%) & 0,66 & 0,25 & 0,34 & 0,55 & 0,35 & 0,12 & 0,09 & 0,61 & 0,16 & 0,09 & 0,45 \\
\hline 4 & Return on Capital Employed & 0,67 & $0,01^{\mathrm{a}}$ & 0,38 & 0,60 & 0,43 & 0,12 & 0,15 & 0,54 & 0,22 & 0,14 & 0,36 \\
\hline 5 & Cash Flow/ Operating Revenue & 0,95 & 0,16 & 0,63 & 0,83 & 0,62 & 0,37 & 0,16 & 0,57 & 0,60 & N/A & 0,17 \\
\hline 6 & Gross Margin $(\%)$ & 0,09 & 0,54 & 0,04 & 0,46 & 0,85 & 0,06 & N/A & 0,87 & 0,12 & 0,23 & 0,09 \\
\hline 7 & EBIT Margin $(\%)$ & 0,96 & 0,95 & 0,53 & 0,65 & 0,20 & 0,12 & 0,22 & 0,84 & 0,12 & 0,11 & 0,81 \\
\hline 8 & EBITDA Margin (\%) & 0,76 & 0,80 & 0,72 & 0,80 & 0,24 & 0,08 & 0,29 & 0,80 & $0,05^{\mathrm{a}}$ & 0,54 & 0,23 \\
\hline 9 & ROE (\%) & 0,52 & $0,01^{\mathrm{a}}$ & 0,44 & 0,49 & 0,51 & 0,29 & 0,15 & 0,41 & 0,58 & 0,67 & 0,08 \\
\hline 10 & ROA (\%) & 0,57 & 0,13 & 0,44 & 0,58 & 0,35 & 0,13 & 0,10 & 0,48 & 0,58 & 0,24 & 0,35 \\
\hline 11 & ROCE (\%) & 0,70 & $0,00^{\mathrm{a}}$ & 0,47 & 0,64 & 0,43 & 0,13 & 0,15 & 0,41 & 0,31 & 0,32 & 0,25 \\
\hline 12 & Current ratio & 0,98 & 0,66 & 0,12 & 0,29 & 0,10 & 0,53 & 0,06 & 0,20 & 0,37 & 0,06 & 0,94 \\
\hline 13 & Liquidity ratio & 0,95 & 0,60 & 0,11 & 0,33 & 0,15 & 0,44 & 0,10 & 0,22 & 0,58 & $0,01^{\mathrm{a}}$ & 0,93 \\
\hline 14 & Interest Cover & 0,28 & 0,49 & 0,24 & 0,65 & 0,13 & 0,07 & $0,02^{\mathrm{a}}$ & 0,78 & $0,04^{\mathrm{a}}$ & 0,27 & N/A \\
\hline 15 & Collection Period (days) & $0,02^{\mathrm{a}}$ & 0,60 & 0,06 & 0,08 & 0,86 & 0,09 & 0,61 & 0,19 & N/A & 0,55 & 0,14 \\
\hline 16 & Credit Period (days) & 0,38 & 0,23 & 0,32 & $0,02^{\mathrm{a}}$ & $0,03^{\mathrm{a}}$ & N/A & 1,00 & 0,37 & 0,18 & 0,13 & 0,15 \\
\hline 17 & Stock Turnover & 0,41 & 0,66 & 0,93 & 0,21 & 0,69 & $0,01^{\mathrm{a}}$ & 0,87 & 0,52 & $0,01^{\mathrm{a}}$ & 0,26 & N/A \\
\hline 18 & Net Assets Turnover & 0,06 & $0,02^{\mathrm{a}}$ & $0,01^{\mathrm{a}}$ & 0,30 & 0,17 & $0,00^{\mathrm{a}}$ & 0,57 & 0,27 & 0,22 & 0,21 & 0,47 \\
\hline 19 & Solvency ratio (\%) & 0,35 & 0,71 & $0,05^{\mathrm{a}}$ & 0,29 & 0,08 & 0,15 & 0,11 & 0,65 & 0,50 & 0,33 & 0,38 \\
\hline 20 & Gearing (\%) & 0,72 & 1,00 & 0,07 & 0,14 & N/A & 0,60 & $0,05^{\mathrm{a}}$ & 0,47 & 0,65 & 0,37 & N/A \\
\hline \multicolumn{2}{|c|}{ Significantly differ } & 1 & 5 & 2 & 1 & 1 & 2 & 2 & 0 & 3 & 1 & 0 \\
\hline \multicolumn{2}{|c|}{ Not significantly differ } & 19 & 15 & 18 & 19 & 18 & 17 & 17 & 20 & 16 & 18 & 17 \\
\hline \multicolumn{2}{|c|}{ Not Available } & 0 & 0 & 0 & 0 & 1 & 1 & 1 & 0 & 1 & 1 & 3 \\
\hline
\end{tabular}

a Significantly differ

Net Assets Turnover is the ratio with three airlines recording significant difference before and after joining the strategic alliance. While other ratios such as profit margin, return on total assets, cash flow/operating revenue, EBIT margin, ROA, current ratio, and solvency ratio have none airline recording significant difference. The average alliance is obtained by excluding companies with no available data, so as not to disturb the entire data.

The low success rate of the strategic alliance in airlines industry is not surprising. In fact, the low performance of alliances is common across other economic sectors [13] despite the wealth of literature in the topic in the last decades. Around 50 percent of alliances does not meet expectations [14]. Thus, researchers are interested in factors affecting alliance performance [15]. The search of key drivers has not converged in many literature, but most focuses on alliance management capability, such as alliance control and strength of ties [13], interorganizational coordination, alliance portfolio coordination, interorganizational learning, alliance proactiveness, and alliance transformation [15]. Low degree of management capability may lead to high failure rate of the alliance, hypothetically including airline alliance. This hypothesis should be tested in future studies.

\section{CONCLUSIONS}

This study indicates that airlines in the alliance on average record a low profitability ratio as can be seen from profit margin at around 5 percent and ROA at approximately 2 percent. A low profitability ratio is caused by the lack of income generated by the aviation industry. Likewise, liquidity ratios are considered low at around 1. A gearing ratio far above 50 percent is typically classified as highly levered or geared. Thus, the company would be at bigger financial risk of default.

Data are tested their normality. More often than not, sample data are drawn from a normally distributed population. Once data are confirmed normally distributed, paired sample t-test are used, otherwise Wilcoxon test are utilized. This study shows the significance level of each test and suggests that in most of financial ratios across airlines, financial difference before and after joining the strategic alliance is not significant.

The low success rate of the strategic alliance in airlines industry is common across other economic sectors. Thus, researchers are interested in factors affecting alliance 
performance. The search of key drivers has not converged in many literature, but most focuses on alliance management capability. Low degree of management capability may lead to high failure rate of the alliance, hypothetically including airline alliance. This hypothesis should be tested in future studies.

\section{REFERENCES}

[1] D. Kartikasari, and P. E. Sanyoto, "Recognition of prior learning breakthrough in aircraft maintenance curriculum". Proceeding of Ocean, Mechanical and Aerospace-Science and Engineering-, Vol.2, 2015 (pp. 1-5). Batam: International Society of Ocean, Mechanical and Aerospace.

[2] D. Elmuti, and Y. Kathawala, "An overview of strategic alliances". Management Decision, 39(3), 2001, pp. 5-218. doi:http://dx.doi.org/10.1108/EUM0000000005452

[3] P. Anslinger, and J. Jenk, "Creating successful alliances". Journal of Business Strategy, 25(2), 2004, pp. 18 - 22. doi:http://dx.doi.org/10.1108/02756660410525362

[4] C. Czipura, and D. R. Jolly, "Global airline alliances: sparking profitability for a troubled industry". Journal of Business Strategy, 28(2), $2007, \quad$ pp. $\quad 57 \quad-\quad 64$. doi:http://dx.doi.org/10.1108/02756660710732666

[5] T. Tyler, IATA Annual Review. International Air Transport Association. $2016 . \quad$ Retrieved https://www.iata.org/publications/Documents/iata-annual-review-16.pdf

[6] T. Flouris, and T. Walker, "Financial comparisons across different business models in the canadian airline industry". Journal of Air Transportation, 12(1), 2007, pp. 25-52.
[7] M. Tsantoulis, and A. Palmer, "Quality convergence in airline co brand alliances". Managing Service Quality: An International Journal, 18(1), 2008, $\quad$ pp. $\quad 34 \quad$ doi:http://dx.doi.org/10.1108/096045810842830

[8] K. Weber, and B. Sparks, "The nexus of strategic alliance research and research on service failure/recovery: advancing conceptual thinking". SERVSIG Conference. Reims, France: American Marketing Association (AMA) . June 12-14, 2003.

[9] K. Weber, "Travelers' perceptions of airline alliance benefits and performance". Journal of Travel Research, 43, 257, 2005. doi:10.1177/00472875042729

[10] S. Harimurti, "Analisis aliansi strategik PT Garuda Indonesia (Persero) Tbk. dan Skyteam". Perpustakaan Pusat UGM, S2 Manajemen. Yogyakarta: Universitas Gadjah Mada. 2017.

[11] Bureau van Dijk Electronic Publishing. Osiris Data Guide. 2007. Retrieved from www.bvdep.com

[12] D. Kartikasari, "Investasi saham aviasi [Investing in aviation stocks]. Haluan Kepri. Batam, Kepri, Indonesia. 2015, January 15th.

[13] O. Mamavi, O. Meier, R. Zerbib, "Alliance management capability: the roles of alliance control and strength of ties", Management Decision, 53 (10), 2015, pp. $2250-2267$.

[14] M. Koza, and A. Lewin, "Managing partnerships and strategic alliances: Raising the odds of success". European Management Journal, 18, 2000, pp. 146-151.

[15] O. Schilke, and A. Goerzen, "Alliance management capability: an investigation of the construct and its measurement", Journal of Management, 36(5), 2010, pp. 1192-1219. 